\title{
Functional MRI for presurgical planning: problems, artefacts, and solution strategies
}

T Krings, M H T Reinges, S Erberich, S Kemeny, V Rohde, U Spetzger, M Korinth, K Willmes, J M Gilsbach, A Thron

\begin{abstract}
Ojectives-Presurgical mapping of motor function is a widely used clinical application of functional (f) MRI, employing the blood oxygenation level dependent contrast. The aim of this study was to report on 3 years experience of 194 fMRI studies on the representation of motor function in 103 patients and to describe the problems and artefacts that were typically present. Methods-An evaluation was carried out to determine whether the patients' age, type or location of the tumourous lesion, severity of the paresis, or the tasks used during the investigation have an effect on artefacts of fMRI studies and how these artefacts are best overcome.

Results-Functional MRI identified the motor regions in $85 \%$ of all investigated paradigms. In $11 \%$ of the investigated patients no information at all on functional localisation was obtained. A draining vein within the central sulcus was present in all patients that showed activation within the parenchyma of the precentral gyrus but also in three patients in whom no parenchymal activation was present. Head movement artefacts were the most frequent cause for fMRI failure, followed by low signal to noise ratio. Motion artefacts were correlated with the degree of paresis and with the functional task. Tasks involving more proximal muscles led to significantly more motion artefacts when compared with tasks that primarily involved distal muscles. Mean MR signal change during task performance was $2.5 \%$.

Conclusions-Most of the artefacts of functional MRI can be reliably detected and at least in part be reduced or eliminated with the help of mathematical algorithms, appropriate pulse sequences and tasks, andprobably most important-by evaluating the fMRI raw data-that is, the MR signal time courses.

(F Neurol Neurosurg Psychiatry 2001;70:749-760)
\end{abstract}

Keywords: functional MRI; presurgical planning; motor cortex

The goal of minimally invasive neurosurgery is to maximise patient safety and to minimise residual tumour. Therefore, the anatomical relation between functional and pathological regions has to be perceived by the neurosurgeon. Traditionally, this has been accomplished by determining anatomical landmarks in relation to the pathological tissue. Because mass effects associated with brain tumours can distort normal cortical anatomy and make the localisation of relevant functional areas by anatomical landmarks alone difficult, preoperative functional localisation is of special interest to the neurosurgeon and ultimately to the patient. Functional MRI (fMRI) combines detailed anatomical information with physiological information on cerebral haemodynamics after cognitive activity to create a structural and functional model of a person's brain. This structural-functional coregistration makes fMRI an ideal tool for presurgical planning of patients with mass lesions affecting functionally important brain regions. Preoperatively, fMRI data can be used for risk management and for determining the operative trajectories - that is, for surgical planning; intraoperatively, fMRI is helpful for guiding surgical resection and for neuronavigation purposes. Individual risks of permanent neurological deficits can be assessed preoperatively and different surgical strategies can be discussed.

The technique of fMRI is easy to employ, even using routine clinical scanners; it is cost effective and yields both structural and anatomical data within the same imaging methodology. Therefore, it has found widespread use over the course of the past several years. Multiple studies have demonstrated the potential of fMRI for presurgical mapping of cortical motor function. ${ }^{1-16}$ All these studies showed the feasibility of fMRI as a non-invasive mapping method for patients with mass lesions in the vicinity of the central sulcus. However, there are only a few studies that focused on problems and artefacts of this technique and on possible solution strategies.

Our intention is to report on our 3 years of experience of 194 functional MRI studies on the representation of motor functions in 103 patients. To the best of our knowledge this is the largest consecutive series of patients investigated with fMRI for presurgical planning purposes. Our aim is to describe the typical problems and artefacts that we have encountered and to provide possible solutions for the clinician. We attempt to elucidate whether functional imaging problems are correlated with the patients' demographic data such as age, type or location of the lesion, severity of the paresis, or the task used during the investigation and to give guidelines how to interpret a functional MRI study. 
Methods

PATIENTS

This was a consecutive series of 103 patients who were investigated with functional MRI between May 1997 and February 2000. The criterion for patient selection was a mass lesion near the central region, distorting normal cortical anatomy by displacing the central sulcus. The patients' age ranged from 9 to 79 years (mean 48.6 years); 59 patients were female, 44 were male. Ninety five patients underwent surgery with resection of the lesion. Lesion localisation was frontal in 49 patients, parietal in 32, and frontoparietal in 12 . The right hemisphere was affected in 42 patients, the left hemisphere in 62 (one patient had a large meningioma of the falx cerebri, affecting both central regions). Symptomatology on admission were motor deficits in 68 patients (including paresis $(n=50)$, loss of dexterity $(n=10)$, or ataxia $(n=8))$, epilepsy in 40 patients (including focal seizures $(n=20)$, focal seizures with secondary generalisation $(n=10)$ and primary generalised seizures $(n=10))$, speech problems in 24 patients (dysarthria $(n=14)$, motor aphasia $(n=8)$, sensory aphasia $(n=2))$, sensory deficits in 20 patients (hypaesthesia $(n=14)$, dysaesthesia $(n=4)$, and paraesthesia $(n=2))$, and nonspecific symptoms in 72 patients (headaches $(n=36)$, nausea $(n=22)$, changes in personality $(n=8))$. Lesion pathology included 23 patients with glioblastoma multiforme, 16 with solitary cerebral metastases, 14 with meningioma, 10 with astrocytoma World Health Organisation (WHO) grade III, and eight with astrocytoma grade II, seven with oligoastrocytoma grade III, four patients each with oligodendroglioma grades II and III, four with a hamartoma, three with an oligoastrocytoma grade II, three with a cavernoma, two with an anaplastic meningioma, and one patient each with a gliosarcoma grade IV, a ganglioglioma, a lymphoma, an arteriovenous malformation, and a calcified aneurysm.

fMRI PROCEDURE

Before entering the scanner, each patient was carefully instructed about the scanning procedure including the possibility of facial muscle contraction due to rapidly switching gradients and the need to avoid head motion. Patient instruction was performed using printouts of typical imaging examples including those with head movement artefacts.

All imaging studies were performed on a 1.5 T Philips Gyroscan NT (Philips Medical Systems, Best, The Netherlands) equipped with echo planar imaging capabilities. The subjects' heads were immobilised in a standard head coil using Velcro straps, neck cushioning, and foam padding to minimise motion artefacts. Before scanning, patients were asked to find a comfortable position in the head coil and to relax during the imaging procedure.

Field homogeneity was optimised for each patient before each scan using an automatic shimming sequence. After localising images in three planes (survey scan), strongly T1 weighted multishot inversion recovery EPI images were obtained for anatomical reference (imaging parameters TR/TE/TI 2000/22/400, FOV $220 \times 220$, matrix $256 \times 256$, slice thickness $7 \mathrm{~mm}$ ). We chose an echo planar imaging technique for the anatomical images instead of a conventional spin echo technique to avoid misregistration of anatomical and functional data due to EPI related distortions. Inversion recovery sequences were chosen to obtain a better grey-white matter differentiation. Images covered the whole brain and were oriented parallel to the line running through the anterior and the posterior commissure. Functional images were obtained using a blood oxygenation level dependent (BOLD) single shot multislice T2* weighted gradient echo EPI sequence (TR/ TE/FA 4000/40/40, FOV 220×220, matrix $64 \times 64$, slice thickness $7 \mathrm{~mm}$ ). In a total scanning time of 4 minutes 24 seconds six alternating epochs of rest and activation each lasting 44 seconds were performed by all patients. Before the functional measurement started, "dummy" scans (acquisitions that were later discarded) were performed by the scanner to approach magnetisation equilibrium. The scan comprised a total volume of 66 time points with a temporal resolution of 4 seconds/ time point. Subsequent to the functional scans the anatomical sequence was repeated after administration of gadolinium DTPA. Each scan (anatomical, functional, and postcontrast anatomical) was obtained with the same slice number, orientation, and localisation.

PARADIGMS

We used boxcar paradigms contrasting motor activation with rest. The motor paradigm was chosen with respect to tumour localisation and motor deficits. Typically, more than one functional paradigm was employed for each patient. Six alternating epochs of rest and activation were performed in all paradigms. During rest periods, patients were asked to relax and to concentrate on the scanner background noise. The patients had sufficient time to practise the task before the scanning session. Performance during the scanning session was controlled via video monitoring. If the patient encountered any problems during the paradigm, the scan was stopped and repeated. Instructions to start and stop were given over the auditory system of the scanner. Before each paradigm was started, the patients were instructed about which movement was to follow. As one video camera was pointed directly at the head of the patient, gross movements of the head were visible while the patient was still in the scanner. If these movements were seen, the scan was aborted, the patient was instructed to refrain from head movements and the scan was repeated.

When investigating the cortical representation of the hand muscles we employed two different paradigms, one of which was performed in every patient: A total of 47 patients underwent a finger opposition paradigm using the affected hand to carry out a self paced light touch of thumb pad to finger pad of each subsequent finger without looking at the hand (finger tapping). Fifty six patients were asked to perform repetitive self paced closing of the 
hand to a fist (hand clenching). In patients with mass lesions affecting the more medial and superior parts of the central area, the lower limbs were tested as well $(n=49)$. The two motor tasks for the lower limbs consisted $(a)$ of repetitive flexion of the toes (toe curling) $(\mathrm{n}=22)$ and $(b)$ of alternating foot flexion and extension (foot flexion) $(\mathrm{n}=27)$. In those patients in whom we tested the facial muscles, we also used two different repetitive tasks, tongue movement from cheek to cheek (tongue movement) $(n=5)$ and contractions of the orbicularis oris muscle (lip puckering) $(n=11)$. As some of the tasks were performed twice in one patient to enhance reliability and some patients performed multiple tasks, a total of 194 functional runs were obtained ( 72 hand clenching, 54 finger tapping, 29 foot flexion, 23 toe curling, five tongue movement, and $11 \mathrm{lip}$ puckering runs).

fMRI ANALYSIS

After image acquisition the data were converted to DICOM standards and transferred via the hospital network to a local network where data evaluation was performed on SUN workstations. Sites of functional activation were statistically evaluated on a voxel by voxel basis using XDS software developed by the Massachusetts General Hospital NMR Center. Using this software, we employed the Kolmogorov-Smirnov non-parametric statistical test to compare the MR signal time course with the given task paradigm. This test was chosen to account for the non-normal distribution of the $\mathrm{T} 2{ }^{\star} \mathrm{MR}$ signal that is presumably due to rhythmic fluctuations (cardiac and respiratory cycle). Transition times (two time points $=8$ seconds after a signal was given) were not included in the statistical evaluation to account for the delayed haemodynamic response curve. The software calculated the activation maps, which were subsequently colour coded on a thermal scale according to the level of statistical significance of the difference between rest and activation states and overlaid over the anatomical inversion recovery EPI images and the contrast enhanced images for anatomical reference and detection of venous structures. Although the low thresholds for activation were set at $p<0.005$ in all cases, the high threshold varied interindividually according to the varying \% signal change between patients. To estimate the noise present in the data, the timecourse of a voxel outside the brain was evaluated for each patient. After calculating the mean MR signal within the selected voxel, the SD of the \% MR signal change was computed. Noise was defined as $2 \times \mathrm{SD}$ and was given in $\% \mathrm{MR}$ signal change units.

SIGNAL TIME COURSE ANALYSIS

Signal time course analysis (calculation of \% change $(\Delta S)$ ) and determination of the latency between onset of task activity and onset of signal rise time $\left(T_{0}\right)$ were performed independently for those voxels that demonstrated task related MR signal changes and that overlaid $(a)$ parenchyma and $(b)$ vessels that were identified on contrast enhanced studies (central sulcal vein and large bridging veins) in all patients and for all tasks. To minimise partial volume effects, only a single voxel was evaluated. We selected this voxel according to its statistical power - that is, the voxel with the peak statistical significance was chosen for further analysis. The mean MR signal intensity for each rest and activation condition of a single functional scan was computed independently. Between each activated state MR signal value and its preceding resting state value the \% signal change was calculated. The \% signal change value was then computed as the mean of the three pairs of rest and activation states. The time of onset of the rise in MR signal intensity was determined as follows. Standard deviation for the baseline MR signal intensity values was calculated. The time of onset $\left(\mathrm{T}_{0}\right)$ was defined as the first time point after beginning the task in which the MR signal intensity was consistently (five consecutive time points) higher than the mean baseline plus 2 SD. A two tailed paired Student's $t$ test was performed to determine whether there were any significant differences in signal time course characteristics ( $\Delta S$ and $T_{0}$ ) between parenchymal and venous "activation". The MR signal time courses were additionally used to differentiate "real" activation from movement related and task related signal changes, again using $\mathrm{T}_{0}$ and $\Delta \mathrm{S}$. If the onset was immediate (starting with the first image during activation states), if it was only present on one (typically the first) start signal, and/or if the signal change exceeded $7 \%$, motion artefact was presumed. To further confirm this, the original $\mathrm{T} 2{ }^{\star}$ raw data of each slice was then visualised as an animated loop that reliably demonstrated variations in head position.

\section{ELIMINATION OF MOTION ARTEFACTS}

If motion artefacts were detected, a motion detection and correction algorithm was used to realign images. ${ }^{17}$ This algorithm depended on the overall measure of similarity between images. If multiple images acquired with the same technique were perfectly aligned to each other, the ratio of one image to the other should be uniform across voxels. Misregistration, on the contrary, would lead to a substantial degree of non-uniformity. The degree of uniformity between images was computed using the SD of the voxel by voxel ratio. The motion correction algorithm iteratively sought to minimise this SD by adjusting the parameters of the rigid body model ${ }^{17}$ This model incorporated a total of six parameters that can be conceptualised as rotations around and translations along each of the three major coordinate axes. To perform rigid body transformations the data were interpolated to cubic voxels before rotation about the three axes and translation within the three planes was possible. The algorithm tried to converge on a reference image (typically the first image of the data set) by computing the ratio of the reoriented image to the reference image on a voxel by voxel basis. The SD served as a measure of the quality of realignment. 
Table 1 Results of functional MRI

\begin{tabular}{lcccccc}
\hline & Hand & Finger & Feet & Toes & Tongue & Lip \\
\hline Number of scans & 72 & 54 & 29 & 23 & 5 & 11 \\
Number of failures & 11 & 4 & 9 & 3 & 2 & 1 \\
Mean \% signal change & 2.7 & 2.8 & 2.4 & 2.2 & 2.6 & 2.5 \\
\hline
\end{tabular}

\section{Results}

FACTORS RELATED TO IMAGING FAILURE

A total of 194 functional scans in 103 patients were performed. Of these, 164 could be evaluated; in 30 runs no functional information was obtained (for details on different paradigms see table 1). In 11 patients, fMRI failed to show activation within the cortical motor regions in all investigated tasks. To evaluate whether specific characteristics of the patients or their pathology correlated with imaging failure, the following factors were investigated: age of the patient, degree of paresis, invasion of the tumour into the precentral gyrus, oedema, displacement of the central sulcus, extravasal blood, and occurrence of abnormal vessels. Although the group of patients with fMR imaging failure was slightly older than the group of patients where fMRI succeeded (52.4 (SD 13.5) years $v 47.2$ (SD 21.3) years) the difference was not significant. Failure to obtain functional information was closely related to motor deficits: in 26 of the 30 failed runs, the patient had a paresis of the investigated limb. For all other investigated characteristics, there was no significant difference between the group of patients in whom fMRI failed and the group in whom fMRI succeeded in showing cortical motor regions.

SIGNAL TO NOISE RATIO IN LARGE DRAINING AND SMALL PARENCHYMAL VEINS

Task related haemodynamic changes after motor activation were visible in various areas. Table 2 shows the occurrence of the significantly different $(p<0.005)$ activated brain areas for all investigated paradigms. Spurious activation, defined as a single significant voxel, was not included in the data. Activation other than spurious activation within a tumour was not seen in any patient. The pattern of activation within the primary sensorimotor cortex after movements of the hand was similar across subjects. We identified a deep medial activity that was located in the "hand knob", ${ }^{18}$ an omega shaped part of the precentral gyrus if the cortical anatomy was not distorted, and, typically in the same slice, more superficial lateral activity within the central sulcus. In subsequent higher slices the lateral activation was still visible on the superficial cortex whereas the medial activity was located maximally on two subsequent slices. The lateral activity was always contiguous on the adjacent slices in all patients, extended towards the superior sagittal sinus, and had a tubule-like structure. When overlaying the functional maps on the contrast enhanced $\mathrm{T} 1$ weighted scan this activation was identified as a large draining vessel. The dual activation visible on at least one slice in 69 patients could also be further differentiated when being merged with the venous anatomy. The lateral more superficial area of activation colocalised in all patients with a vessel seen after administration of Gd-DTPA whereas the medial deep activation did not spatially coincide with a vessel seen on contrast enhanced studies but did instead overlay the parenchyma (fig 1 , a and b). Per cent T2 $2^{\star}$ signal changes between rest and activation states $(\Delta S)$ were calculated in parenchymal voxels and voxels overlaying draining veins for each patient and for every task. Mean parenchymal \% MR signal change between rest and activation states was $2.5 \%$ (SD $0.8 \%$, minimum $0 \%$ and maximum $4.3 \%$ ) for all investigated paradigms (for details on the different paradigms see table 1). Significant haemodynamic changes in large draining veins occurred irrespective of the motor paradigm used. Mean \% changes in signal intensity within the draining veins were typically 2.4 times (SD 0.54: minimum 1, maximum 4.4) as high as the increase within the parenchyma (see fig 2 and fig 3 for an example) (mean \% signal change within draining veins: $5.8 \%$ (SD $1.6 \%$, minimum $0 \%$, maximum: $9.6 \%$ ). $t$ Test statistics of the time course analysis disclosed highly significant $(p<0.001)$ differences in $\Delta S$ and nonsignificant differences in $T_{0}$ between parenchymal and venous sites of task related haemodynamic changes. $T_{0}$ in large draining veins and parenchymal venules coincided in most cases using the rather coarse time resolution of 4 seconds (TR $4000 \mathrm{~ms}$ ). Mean $\Delta \mathrm{S}$, SD of $\Delta \mathrm{S}$, the range of $\Delta S$, and the correlation between venous and parenchymal $\Delta S$ in both large draining vessels and small parenchymal venules of all investigated subjects are given in table 3. In figure $1 \mathrm{c}$, representative time course examples during finger opposition for small parenchymal venules and a large draining vein are shown.

Significant activation within large venous structures could be seen up to $2.8 \mathrm{~cm}$ away from the site of parenchymal activation, in some cases even within the superior sagittal sinus. Mean distance between the site of parenchymal activation and the venous activation most distant from the parenchymal site was $1.9 \mathrm{~cm}(\mathrm{SD} 1.1 \mathrm{~cm})$. The mean distance between the peak parenchymal voxel and the peak venous voxel was $1.0 \mathrm{~cm}$ (SD $0.8 \mathrm{~cm}$ ). The number of statistically significant voxels

Table 2 Activated brain regions during functional MRI $(p<0.005)$

\begin{tabular}{|c|c|c|c|c|c|c|}
\hline & Hand & Finger & Feet & Toes & Tongue & Lip \\
\hline Primary motor cortex (M1), contralateral & 61 & 50 & 20 & 20 & 3 & 10 \\
\hline Primary motor cortex (M1), ipsilateral & 17 & 21 & 7 & 4 & 3 & 10 \\
\hline Primary sensory area (S1) & 39 & 33 & 4 & 1 & 0 & 2 \\
\hline Supplementary motor area & 35 & 24 & 12 & 11 & 1 & 5 \\
\hline Premotor areas & 14 & 17 & 3 & 6 & 0 & 3 \\
\hline Cerebellum & 56 & 44 & 13 & 15 & 2 & 7 \\
\hline
\end{tabular}




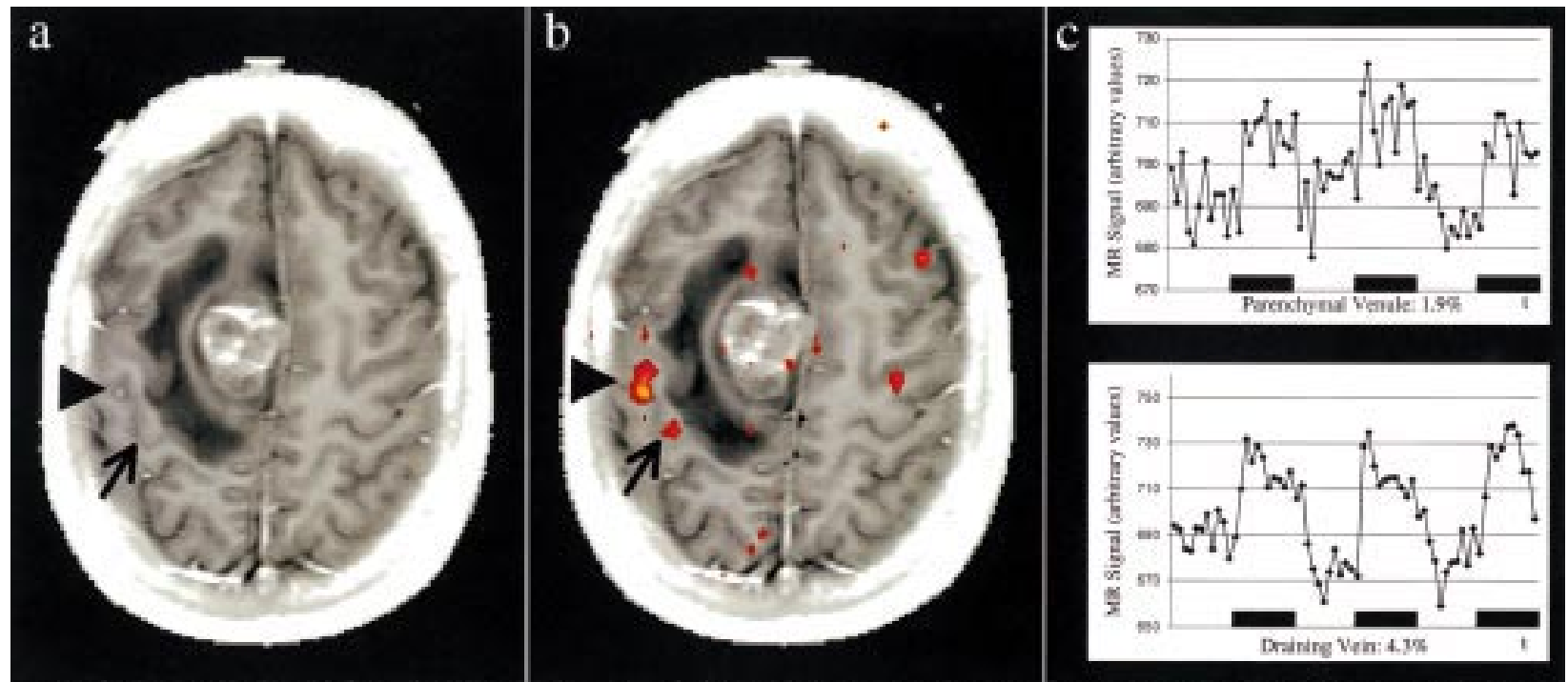

Figure 1 Detection of venous activation using contrast enhancement. This patient with a metastasis performed a left finger tapping task during functional imaging. Frames $a$ and $b$ : In the right central region there is dual activation: a medial activation (arrow) and a more lateral activation (arrowhead). This lateral activation overlies the central sulcal vein that is identified on the contrast enhanced anatomical scan (a), whereas the medial activation overlies the parenchyma of the precentral gyrus. Frame c: time courses of the large draining vein (arrowheads on frames a and b) and small parenchymal venules (arrows on frames a and b). With a coarse temporal resolution (4 s/image), no significant differences in the time to onset of the haemodynamic changes can be identified. The size of activation, its significance, and the \% signal change within the larger vein are more prominent compared with small parenchymal venules.

$(\mathrm{p}<0.005)$ was always larger around draining veins (mean 17 voxels (SD 11.6 voxels) compared with parenchymal venules (mean 6 voxels (SD 8.3 voxels) (for an example see fig 1 b). In seven functional runs (two hand clenching runs, two finger tapping runs, two foot flexion, and one toe curling run), no parenchymal activation was seen within cortical motor areas. Low signal to noise ratio was therefore held responsible for seven of the 30 failed fMRI examinations. In three of these seven runs, no parenchymal but draining vein activation was seen (for an example see fig 4). Noise within the data was $1.2 \%$ (SD $0,24 \%$ ) minimum $0.7 \%$, maximum $1.9 \%$ ) and was not related to the investigated paradigm.

MOTION ARTEFACTS

Motion related artefacts were present in 39 of the 72 hand clenching paradigms and made an interpretation impossible in nine of these functional runs (72/39/9); during finger tapping the

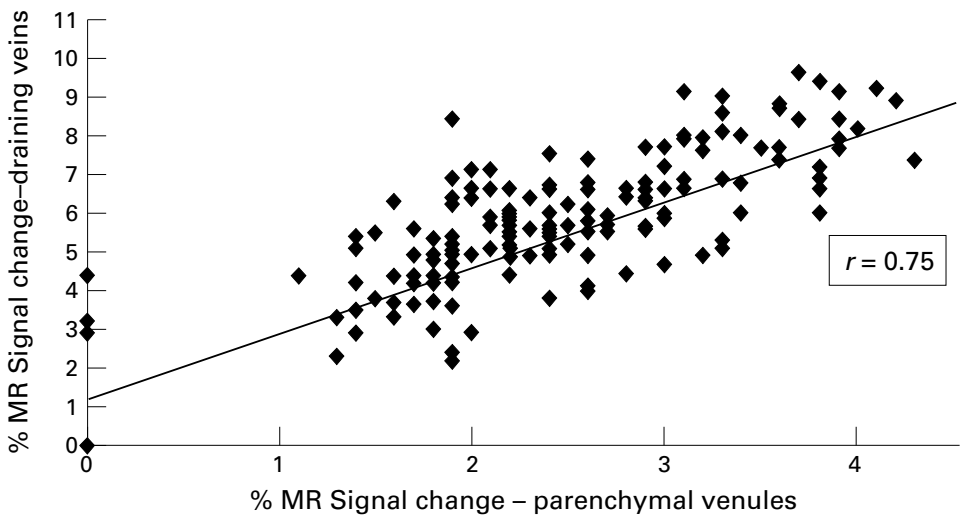

Figure 2 Mean \% MR signal change in large draining veins versus small parenchymal venules. Each diamond represents a pair of $\% M R$ signal changes within large veins and small venules for all investigated functional scans. Per cent signal change in larger veins is typically 2.4 times as high as in parenchymal venules. In some patients, no parenchymal activation was visible whereas activation in larger veins was still evident (see fig 3 for an example). ratios were 54/16/2, during foot flexion 29/ $17 / 7$, and during toe curling $23 / 8 / 2$. Lip puckering had ratios of $11 / 8 / 1$ runs, tongue movement $5 / 5 / 2$. Motion related artefacts were therefore responsible for 23 of the 30 failed fMRI examinations. The frequency of motion related artefacts was related to the degree of paresis (fig 5). If no paresis of the investigated muscle was present, motion artefacts were present in $29 \%$, in patients with paresis $72 \%$. In those patients who did not have a paresis motion artefacts occurred more often during tasks involving proximal muscles (hand clenching $30.3 \% v$ finger tapping $1.8 \%$, foot flexion $38.5 \% v$ toe curling $18.2 \%$ ). There were no significant relations between the occurrence of motion artefacts and patient age, sex, or type of lesion. In the parenchymal areas, the latency between task commencement and first significant increase in MR signal was not immediately visible in any patient $\left(\mathrm{T}_{0}=0: 0 \%, \mathrm{~T}_{0}=1\right.$ : $0 \%)$, The MR signal increase started between 4 and 12 seconds after task commencement $\left(\mathrm{T}_{0}\right.$ $=2: 47 \%, \mathrm{~T}_{0}=3: 30 \%, \mathrm{~T}_{0}=4: 21 \%$ ); only sporadically, the increase started later than 12 seconds $(2 \%)$. For motion artefacts that were detected by the typical ring-like appearance around the outer rim of the skull (halo artefact) or by inspecting the animated loop of T2 images, a significant increase in MR signal most often began immediately after task commencement $\left(\mathrm{T}_{0}=0: 24 \%, \mathrm{~T}_{0}=1: 46 \%\right)$. At $\mathrm{T}_{0}=2-4$ only $7 \%$ of the functional runs demonstrated significant MR signal increase; the remaining $23 \%$ were equally distributed over $\mathrm{T}_{0}=5-10$ (for examples of time courses with abrupt head movement see fig $6 \mathrm{~A}$ and $\mathrm{B}$ ). In addition to $T_{0}$ computation, $\Delta S$ of motion artefacts was evaluated. Mean \% signal change was $7.7 \%$ with a range from $1.4 \%$ to $27.3 \%$ and $\mathrm{SD}$ of $16.5 \%$. In patients in whom ring-like artefacts were present and in those in whom running an animated loop through the image 


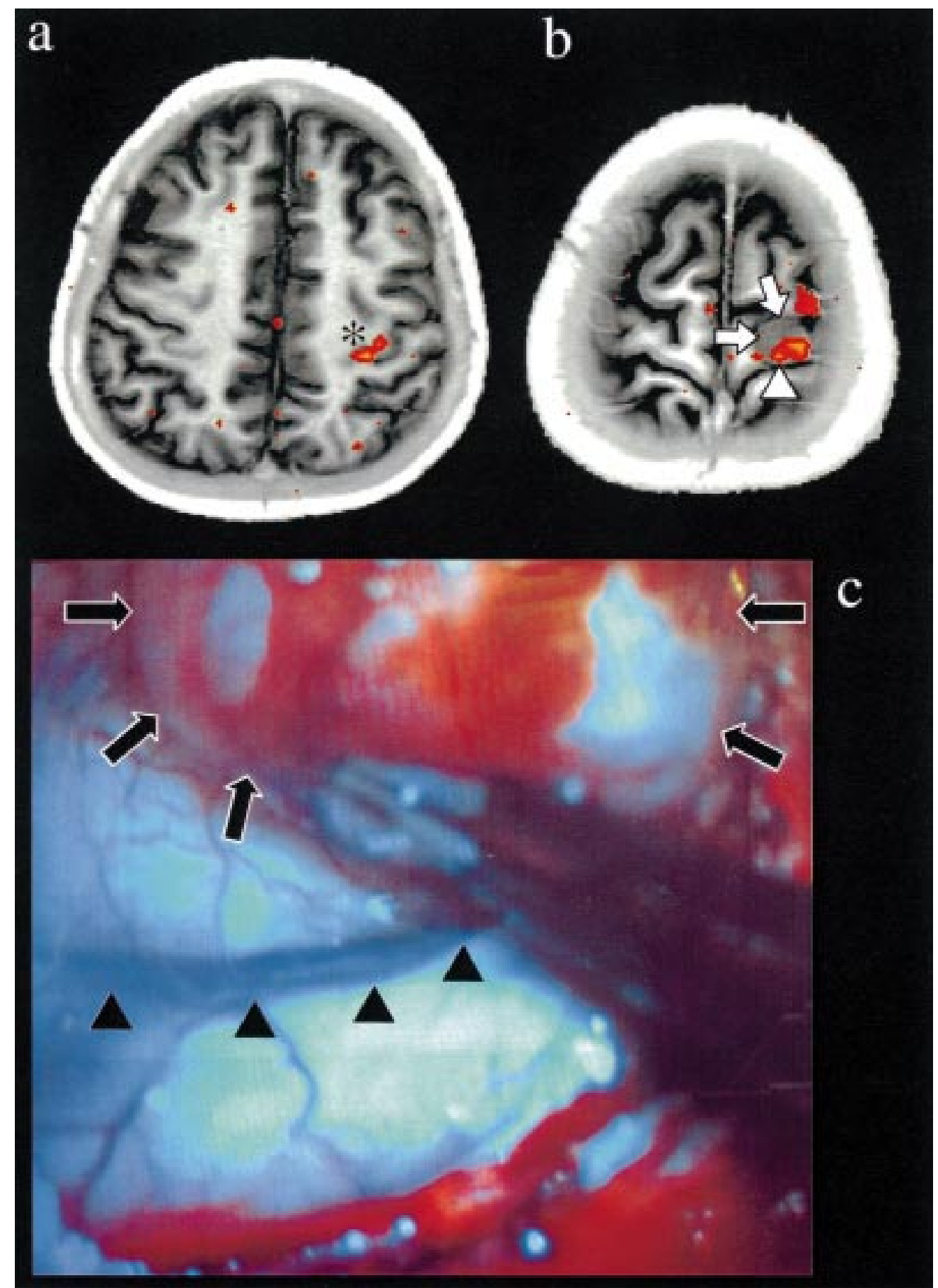

Figure 3 Intraoperative demonstration of the large draining vein visible during $f M R$ examination. Notice the parenchymal activation (* in a) that was located $15 \mathrm{~mm}$ below the most significant activation (white arrowhead in b); in this patient with a convexity meningioma (arrows in $b$ and $c$ ). The most significant activation was directly adjacent to the meningioma. In the operative site note the large central sulcal draining vein (black arrowheads in (c) that was the anatomical correlate of the functional "activation".

Table 3 Differences between large draining veins and small parenchymal venules

\begin{tabular}{|c|c|c|c|c|c|c|}
\hline & Hand & Finger & Feet & Toes & Tongue & Lip \\
\hline \multicolumn{7}{|l|}{ Parenchymal venules: } \\
\hline$\%$ Signal change & 2.7 & 2.8 & 2.4 & 2.2 & 2.6 & 2.5 \\
\hline SD & 0.8 & 0.8 & 0.6 & 0.4 & 1.0 & 0.7 \\
\hline Minimum & 0 & 0 & 0 & 0 & 1.4 & 1.6 \\
\hline Maximum & 4.2 & 4.3 & 3.6 & 2.9 & 3.3 & 3.8 \\
\hline \multicolumn{7}{|l|}{ Large draining veins: } \\
\hline$\%$ Signal change & 6.3 & 6.3 & 6.1 & 4.9 & 4.3 & 5.6 \\
\hline SD & 1.5 & 1.7 & 1.2 & 1.1 & 1.3 & 0.9 \\
\hline Minimum & 0 & 0 & 0 & 0 & 2.9 & 4.4 \\
\hline Maximum & 9.6 & 9.2 & 8.4 & 6.6 & 5.3 & 6.6 \\
\hline Correlation coefficient $(r)$ & 0.80 & 0.81 & 0.38 & 0.57 & 0.99 & 0.62 \\
\hline
\end{tabular}

raw data showed head movement, an automated motion detection and correction algorithm was employed ( $\mathrm{n}=93$ runs). In 23 functional runs, no improvement was noted. The remaining 70 runs did show improvement both because of eliminated motion artefacts and in some cases, also because of an enhanced parenchymal signal (fig 7). In the group of patients in whom the motion correction algorithm failed, head movements in all three directions with movements exceeding $2 \mathrm{~mm}$ 


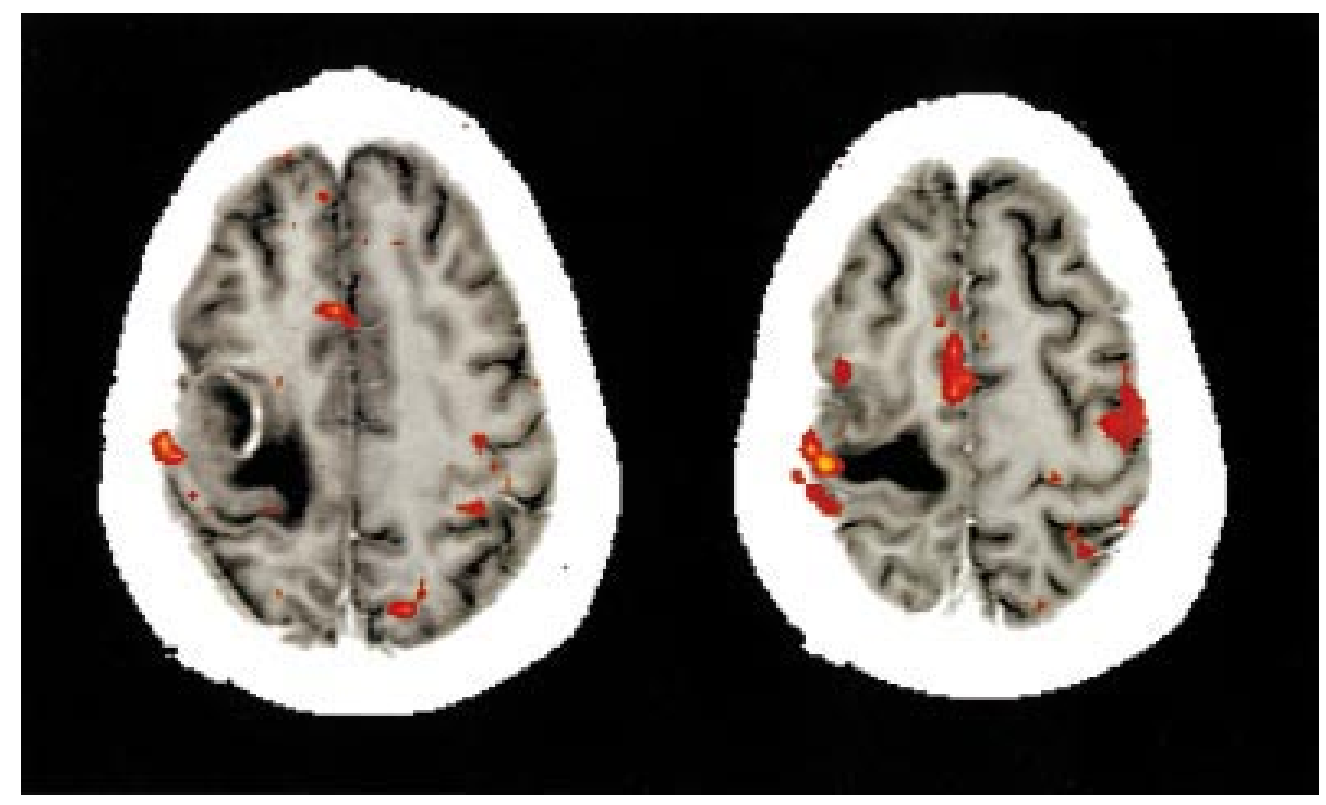

Figure 4 This patient with a solitary metastasis does not show any parenchymal activation whereas activation within the vein draining the central region is still visible. Per cent signal change between rest and activation within the parenchyma might be too low to yield significant results whereas activation within the draining veins with its higher \% signal change can still be detected. In this patient, additional activation within premotor regions, the supplementary motor area, and the ipsilateral motor cortex can be seen.

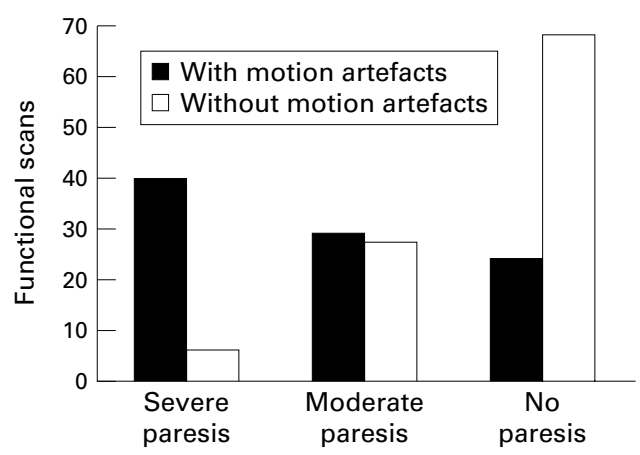

Figure 5 Degree of paresis $v$ degree of motion artefacts. The height of the black bars indicates the number of functional scans in which motion artefacts were present, the open bars represent scans without motion artefact. In patients with a paresis, motion artefacts occur more often, presumably due to coactivation of proximal muscles.

were noted. In those in whom motion correction succeeded the movement was predominantly noted in one direction, and was usually smaller than $1.5 \mathrm{~mm}$.

\section{Discussion}

Preoperative assessment of motor function can be performed with various methods in addition to fMRI. Positron emission tomography (PET) has been used since $1987 . .^{19}$ One of the main advantages of PET over fMRI is better signal to noise ratio. However, PET facilities are not as widely available as MR facilities, imaging is less cost effective, and the spatial resolution is inferior. $^{3}$ Coregistration of functional PET data with anatomical MRI or CT data is still necessary. Finally, fMRI is not dependent on radiation, making it more suitable for routine presurgical evaluation. Dipole source analysis of magnetoencephalographic $(\mathrm{MEG})^{20}$ and electroencephalographic (EEG) data ${ }^{21}$ have also been used for presurgical planning. Both methods have an excellent temporal resolution and can localise the central sulcus with high accuracy. However, by contrast with fMRI, these methods are again more time consuming and not widely available. The major drawback, however, is that both EEG and MEG dipole source algorithms determine a point source and not an extended field of activated brain tissue. ${ }^{22}$ By means of MEG and EEG alone the main goal of a minimal invasive approach - to spare all regions relevant for a specific function-cannot be assessed preoperatively. Recently, transcranial magnetic stimulation (TMS) in conjunction with a neuronavigation system (stereotactic TMS (STMS)) has demonstrated its potential as an easy to use and cost effective method for preoperative assessment of cortical areas involved in motor execution. ${ }^{23}$ However, so far only motor functions can be reliably mapped and the exact localisation accuracy is not yet determined in a larger series of patients.

Although fMRI seems to be the method of choice for the preoperative evaluation of patients with mass lesions near functionally important brain regions, ${ }^{1-16}$ no study has so far focused on the potential problems and artefacts of this technique. Therefore we discuss these problems and the possible solution strategies that we employed.

\section{SIGNAL FROM LARGER VESSELS}

Functional MRI depicts neuronal activity indirectly via haemodynamic changes in the human brain. Vessels draining the site of neuronal activation, not the active neurons, are disclosed. By contrast with small draining veins in the brain parenchyma (microvasculature) the larger draining veins (macrovasculature) have an unclear spatial relation to the actual site of neuronal activation. Detection of functional MR activation in these vessels is therefore less desirable. The so-called "brain or vein" 
problem $^{24}$ characterises the lack of knowledge about whether large draining veins without a close relation with the areas of cerebral activation or small parenchymal venules in close proximity to the sites of neuronal activity (maximally $1.5 \mathrm{~mm}$ apart) are identified during BOLD fMRI. ${ }^{25}$
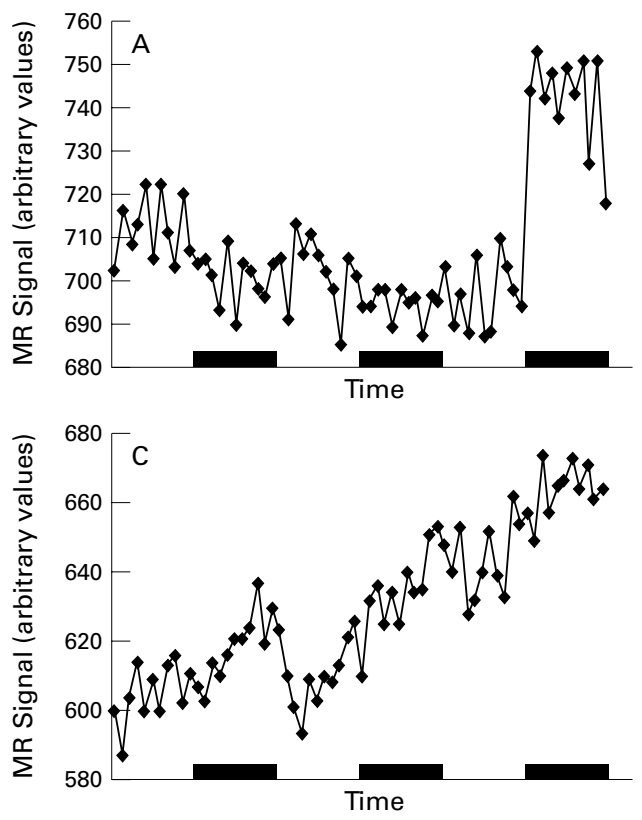

Figure 6 MR signal time courses during different types of movement artefacts. (A) is an MR signal time course of a functional scan during which an abrupt movement occurred during task commencement in the third "on period". The increase in MR signal is so pronounced that a significant "activation" is computed for the cortical area this time course stems from. (B) shows a reverse example: in this cortical area task related haemodynamic changes were seen (note the relative increase in the first and third "on period"). However, during the second "on period" a large decrease in MR signal secondary to an abrupt head movement prevented this area from showing a significant activation. In (C) a time course with a gradual increase in MR signal secondary to slow head movements is demonstrated. This type of artefact might mimic activation because the MR signal within each "on period" is higher than in every preceding "off period". (D) shows an example of a cortical area with task related haemodynamic changes that are obscured by gradual head movements. Motion correction would lead to an increase in statistical significance within this area.
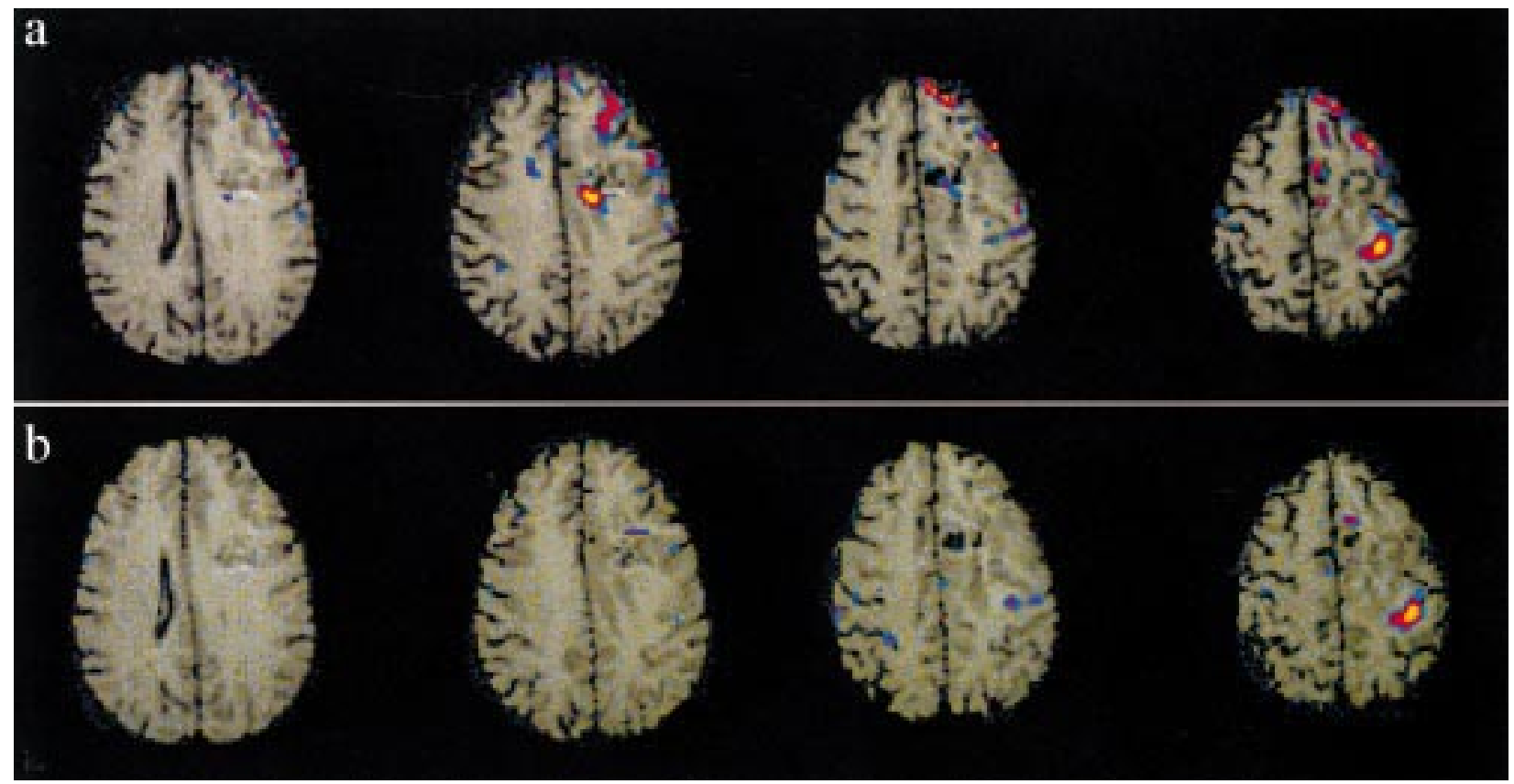

Figure 7 (a) Movement related artefacts. This frame shows a patient with a left frontal arteriovenous malformation that was hyperintense on T2* weighted BOLD images during clenching of her right hand. Due to movement related artefacts in the superior-inferior direction, this area of high signal intensity was shifted during task performance into areas of lower T2* signal intensity, mimicking a task related increase in the BOLD signal and therefore "activation" within the tumour. (b) Motion artefact reduction using mathematical algorithms. This frame shows the same slices as in (a) after mathematical motion correction using identical statistical threshold levels. The false activation within the lesion and the left frontal halo are extinguished, whereas activation within $M 1$ remains unchanged. 
and anterior banks of the precentral and postcentral gyrus ${ }^{26}$ are visible after contrast administration. In $88 \%$ of all investigated paradigms, we found superficial activation within the central sulcus after motor activation, in $86 \%$ of the functional runs an additional deep medial area overlaying the parenchyma of the precentral gyrus was found. In all patients in whom a deep medial activation was seen, we also found the superficial more lateral area of task related haemodynamic changes. The lateral area of activation overlaid a vein visible on contrast enhanced T1 weighted sequences whereas the medial area did not. Moreover, the lateral area extended over multiple slices, being a tubulelike structure. This activation, therefore, reflects a superficial vein within the central sulcus, draining primarily upwards, or in axial slices, lateral, towards the brain surface and the superior sagittal sinus, whereas the medial activation reflects parenchymal microvasculature. Merging of functional images with contrast enhanced $\mathrm{T} 1$ weighted images can therefore differentiate functional MR signal changes of large draining vessels from those of small parenchymal vessels. ${ }^{27}$

SIGNAL FROM LARGER VESSELS: SOLUTIONS FROM DIFFERENT PULSE SEQUENCES

Monte Carlo modelling of susceptibility physics has shown that unlike spin echo sequences, gradient echo sequences are not specific in the detection of vessel sizes. ${ }^{28-30}$ Both macrovessels and microvessels are detected with a high sensitivity. Spin echo sequences on the other hand, have a high specificity for microvessels but are less sensitive to magnetic susceptibility effects. At $1.5 \mathrm{~T}$, functional contrast for both sequences is similar within parenchymal regions whereas signal changes in large vessels are greatly reduced by employing an additional refocusing pulse as used in spin echo sequences. ${ }^{31}$ The diminution of signal arising from large vessels is not seen for gradient echo sequences. This decreased specificity for detected vessel size of gradient echo sequences is traded against a higher overall sensitivity for magnetic susceptibility differences and therefore a higher $\%$ signal change in gradient echo sequences compared with spin echo sequences. Therefore, we think that whenever possible $\mathrm{T} 2{ }^{\star}$ weighted spin echo sequences-for example, asymmetric spin echo sequences ${ }^{31}$ - should be employed in presurgical evaluation of motor function.

SIGNAL FROM LARGER VESSELS: SOLUTIONS FROM TIME COURSE DATA

The MR signal time courses can provide valuable data for discerning large draining veins from parenchymal venules both in regard to time to onset of the increase in MR signal and the \% signal change. Differences in the onset of signal change between large draining veins and cortical microvasculature have been reported previously. $^{32}$ It has been shown that the increases in MR signal during task performance in large draining veins are time delayed compared with those from the parenchymal microvasculature corresponding to the sites of neuronal activity. These authors argued that the longer delay was consistent with the time required for blood to pass smaller veins and reach larger vessels. The differences for macrovasculature and microvasculature in the rise time was 1-3 seconds. A time resolution of 4 seconds, as was used in our fMRI experiments, is therefore too coarse to detect robust differences between both types of activation. Similarly, the finding of an initial dip of the BOLD MR signal corresponding to an early rise in deoxyhaemoglobin seen by spectroscopic imaging ${ }^{33}$ as an alternative approach to discern larger draining veins from parenchymal activation is not possible with this time resolution. This initial dip, which is only seen in small venules with a close spatial correspondence to the neuronal activity, occurs in the order of $100-300 \mathrm{~ms}$ after a stimulus is presented and therefore is not detectable with the routine functional imaging sequences.

We have found a considerably higher \% signal change in large draining veins compared with parenchymal venules, which has also been reported previously. ${ }^{34}{ }^{35}$ These differences in \% BOLD MR signal intensity changes seen in the described gradient echo pulse sequence can be explained by different effects. Firstly, magnetic susceptibility effects and a concomitant increase in \% signal change are enhanced around large veins draining an extended activated region. ${ }^{27}$ Secondly, the macroscopic signal increase during neuronal activation reflects a net increase in brain oxygenation within an extended field of brain tissue. The more prominent increase in \% signal change might therefore be due to a partial volume effect. As parenchymal vessels are considerably smaller than the draining macrovessels, parenchymal activation suffers from larger partial volume averaging with brain tissue unaffected by the motor activation. Thirdly, inflow effects, which have a significant impact on $\%$ signal changes $^{24}{ }^{36}$ are less pronounced in cortical microvasculature because there is a random distribution of capillary orientation. ${ }^{37}$ Fourthly, $\%$ signal changes might also be larger in the macrovasculature as the venous drainage in larger sulcal vessels have contributions from a larger area-that is, both the precentral and postcentral gyrus - which are both active during motor tasks.

Because large draining veins with an unclear relation with the site of neuronal activity have larger \% signal changes, caution must be taken if a study shows a low signal to noise ratio. Parenchymal activation yields relatively lower $\%$ signal changes (typically in the order of $2.5 \%$ but in some patients as low as $1.6 \%$ ). If background noise (typically in the order of $1.2 \%$ but in some studies as high as $1.9 \%$ ) approximates to the parenchymal activation \% signal change, this change might be too low to yield significant results. ${ }^{38}$ In these cases parenchymal activation with a lower statistical power might even be non-detectable, whereas venous activation still is, which leads to misinterpretation of the anatomical correlate of the specific task related activity as only larger venous structures demonstrate a high enough $\Delta S$ and 


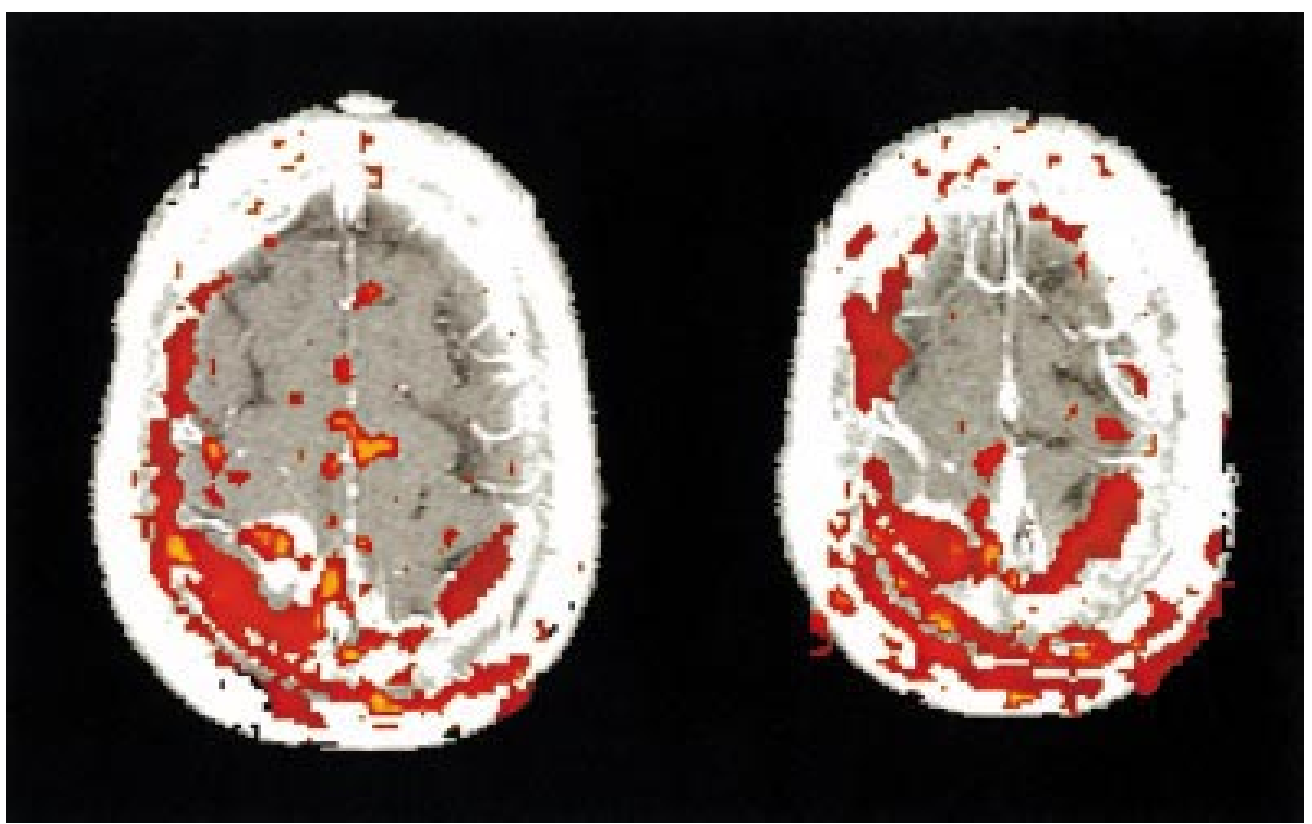

Figure 8 Movement related artefacts. This figure shows the typical motion artefact present during movements in the axial plane (from left to right) appearing as a "halo" around the brain. This patient with a severe paresis of her left upper limb caused by a glioblastoma multiforme showed head movements exceeding $1 \mathrm{~cm}$ in the axial plane during task performance that could not be corrected by mathematical algorithms.

therefore a high enough statistical power. This might explain why, in three patients, we did not find parenchymal activation whereas we did see the lateral draining vein (see fig 3 for an example). Here, the low signal to noise ratio of fMRI might lead to misinterpretation of the real location of motor activity. When evaluating higher cognitive functions such as preoperative language mapping caution must be taken as for these paradigms $\Delta S$ is even lower; therefore, active parenchyma will more often demonstrate \% MR signal changes too low to be detectable.

MOVEMENT RELATED ARTEFACTS

Head movement related artefacts are present when voxels of higher $\mathrm{T} 2{ }^{\star}$ signal intensity are shifted into positions formerly occupied by voxels with lower MR signal intensity. Therefore, regions with high intensity gradients across neighbouring voxels are particularly prone to movement related artefacts. Local susceptibility artefacts are present at regions at the base of the brain, next to the orbits, near the petrous bones and the frontal sinuses, near the outer rim of the brain and skull, the ventricles, and even near tumour tissue (fig 6 A). Highly vascularised tumours such as arteriovenous malformations, tumours containing extracellular methaemoglobin (cavernous haemangioma), and tumours with surrounding oedema have a high signal on $\mathrm{T} 2{ }^{\star}$ weighted sequences, tumours containing haemosiderin, melanomas, or tumours containing large amounts of protein or fat on the other hand, are typically hypointense on $\mathrm{T} 2{ }^{\star}$ weighted sequences compared with brain parenchyma. In some of the aforementioned areas, MR signal intensity gradients of up to $50 \%$ can be seen..$^{39}$ Here, even a movement in the order of $1 / 20$ of a voxel length $(0.2 \mathrm{~mm})$ can lead to significant MR signal changes that may erroneously be interpreted as activation. Particularly in tumours infiltrating the motor areas, identification of these artefacts is paramount for surgical planning. Depending on the type of movement (for example, movements in the axial plane; torsional head movement in the coronal plane, tilting of the head in the sagittal plane), these artefacts may occur at different borders of the structures mentioned. Motion in the axial plane typically has a ring-like spatial appearance around the respective brain structures after statistical evaluation (fig 8). Hajnal et al ${ }^{40}$ reported that sometimes these types of motion artefacts cannot be differentiated from "real" activation, as they typically lead to higher \% signal changes and, therefore, to a higher statistical significance.

We found a high degree of correlation between motion related artefacts and degree of paresis. If a paresis was present, motion artefacts occurred significantly more often than in patients without a paresis $(72 \% v$ $29 \%$ ). In patients with paresis, the proximal extremity musculature is typically still intact as proximal and trunk muscles receive input from both hemispheres. To perform the required task, the patient therefore often showed coinnervations of the shoulder muscles for tasks involving the upper limb and with muscles of the trunk in tasks involving the lower limb. Typically, both types of compensatory movements additionally lead to head movements or, even worse, to movements of the head coil if not properly fixed.

Head movement does not only induce additional "false" activation, it can also diminish or even mask "real" activation by changing the signal time course in such a way that the task synchronous MR signal is obscured. Both abrupt and gradual head movements can cause this effect. Abrupt head movements lead to abrupt changes in signal time course (fig 6 A). 
If an abrupt head movement leads to a sudden decrease of the MR signal time course during task activation, statistical tests will not show the affected voxels to be "active" as no task related increase in MR signal is obvious (fig $6 \mathrm{~B}$ ). Gradual head movements (fig $6 \mathrm{C}$ and $\mathrm{D}$ ) might have the same effect.

THE MOTION ARTEFACT PROBLEM: SOLUTIONS FROM CHOOSING THE RIGHT PARADIGM

In patients with a paresis, different paradigms can be used to avoid artefacts while maintaining functional information. Possible paradigms are sensory stimulation, passive movements, or motor imagery. It might also be useful in patients with a severe paresis to consider alternative mapping modalities mentioned above (TMS, PET). In patients without a paresis, hand clenching resulted in motion artefacts in $30.3 \%$, whereas only $14.8 \%$ of the patients without a paresis showed movement related artefacts during finger tapping. The same was true for muscles of the lower limb; foot flexion resulted in motion artefacts in $38.5 \%$ of the scans, toe curling only in $18.2 \%$. In patients without impairing neurological deficits, tasks involving more distal muscles should therefore be favoured against those tasks involving additional proximal muscles. These tasks activate the hand and foot area reliably and with a lesser degree of movement related artefacts. As more distal muscles are represented over a larger cortical region than proximal muscles, only a minimal loss of \% signal intensity is theoretically expected and was in fact found in our experiments.

THE MOTION ARTEFACT PROBLEM: SOLUTIONS FROM TIME COURSE DATA

The BOLD response curve after neuronal activation demonstrates the first $\mathrm{T} 2^{\star}$ signal increase about 4 seconds after commencing the task with the peak (mean $2.5 \%$, maximum $4.3 \%$ ) reached at about 8 seconds. ${ }^{38}$ Movement related artefactual activation does not follow these considerations. When starting the task, the MR signal increases abruptly and reaches its maximum directly and without a delay. There is no gap between the start of the task and MR signal increase and no gradual increase of the MR signal to reach a maximum. An initial dip that is found when scanning with a high temporal resolution within the parenchymal vessels and that corresponds to the initial decrease in oxyhaemoglobin content due to the delayed increase in regional cerebrovascular blood flow and volume ${ }^{33}$ is never seen in the raw MR data of motion artefacts. Per cent signal changes during head movements are typically higher than $5 \%$. Concurring with the increase in MR signal on one border of high tissue susceptibility gradients is a mirrored decrement of MR signal on the opposite border (surrounding voxels or the other side of the head) that is, depending on the statistical test used, not necessarily visualised. Time course analysis can therefore be reliably used for the detection of head motion artefacts.
THE MOTION ARTEFACT PROBLEM: SOLUTIONS FROM MATHEMATICAL ALGORITHMS

Before starting data evaluation, functional scans should be scanned for interimage motion artefacts by running an animated loop through the whole set of images. Gross head movements should be avoided during image acquisition; however, as mentioned above, even motion in the subvoxel magnitude can lead to severe artefacts in the vicinity of tissue boundaries. Although we did not employ motion correction on a regular basis we think that motion correction is worth the additional time during data evaluation and should be employed on a regular basis. Now it is our policy to perform automated motion correction in all patients, regardless whether motion artefacts are present or not. Advantages of the algorithm we used in our study are that it is not dependent on a large field of view, a multiple slice study, or a particular starting position. Compared with other alternative registration techniques this approach has performed favourably both in speed and accuracy. ${ }^{41}$

\section{Conclusion}

This study includes the largest number of patients investigated with functional MRI for presurgical planning so far. We have shown that problems and artefacts of functional MRI can be reliably detected and at least in part reduced or eliminated with the help of mathematical algorithms, appropriate pulse sequences, and task paradigms; and-in our opinion most important-by evaluating the fMRI raw data (the MR signal time courses). Uncritical use of fMRI and belief in all "bright spots" on a functional imaging study should be avoided. We conclude that functional MRI has to be checked carefully for different types of artefacts before it can be used routinely in the presurgical decision making process. Despite the promising potentials of functional MRI (spatial and temporal resolution, cost effectiveness, ease of use) proper interpretation of the results requires experienced colleagues, just as with any other neuroradiological procedure.

1 Jack CR Jr, Thompson RM, Butts RK, et al. Sensory motor cortex: correlation of presurgical mapping with functional MR imaging and invasive cortical mapping. Radiology 1994;190:85-92.

2 Puce A, Constable RT, Luby ML, et al. Functional magnetic resonance imaging of sensory and motor cortex: comparison with electrophysiological localization. $f$ Neurosurg 1995;83:262-70.

3 Cosgrove GR, Buchbinder BR, Jiang H. Functional magnetic resonance imaging for intracranial navigation. magnetic resonance imaging for intac

4 Yousry TA, Schmid UD, Schmidt D, et al. The central sulcal vein: a landmark for identification of the central sulcus cal vein: a landmark for identification of the central sulcus
using functional magnetic resonance imaging. $f$ Neurosurg 996 Oct; 85:608-617.

5 Roux FE, Ranjeva JP, Boulanouar K, et al. Motor functional MRI for presurgical evaluation of cerebral tumours. Stereotact Funct Neurosurg 1997;68:106-111.

6 Stapleton SR, Kiriakopoulos E, Mikulis D, et al. Combined utility of functional MRI, cortical mapping, and frameless stereotaxy in the resection of lesions in eloquent areas of brain in children. Pediatr Neurosurg 1997;26:68-82.

7 Dymarkowski S, Sunaert S, Van Oostende S, et al. Functional MRI of the brain: localisation of eloquent cortex in focal brain lesion therapy. Eur Radiol 1998;8:157380.

8 Krings T, Reul J, Spetzger U, et al. Functional magnetic resonance mapping of sensory motor cortex for imageguided neurosurgical intervention. Acta Neurochir (Wien) 1998; 140:215-22.

9 Nitschke MF, Melchert UH, Hahn C, et al. Preoperative functional magnetic resonance imaging (fMRI) of the 
motor system in patients with tumours in the parietal lobe. Acta Neurochir (Wien) 1998;140:1223-9.

10 Pujol J, Conesa G, Deus J, et al. Clinical application of functional magnetic resonance imaging in presurgical identification of the central sulcus. F Neurosurg 1998;88:863-9.

11 Schulder M, Maldjian JA, Liu WC, et al. Functional imageguided surgery of intracranial tumours located in or near the sensorimotor cortex. F Neurosurg 1998;89:412-18.

12 Wildforster U, Falk A, Harders A. Operative approach due to results of functional magnetic resonance imaging in central brain tumours. Comput Aided Surg 1998;3:162-165.

13 Achten E, Jackson GD, Cameron JA, et al. Presurgical evaluation of the motor hand area with functional MR imaging in patients with tumours and dysplastic lesions. Radiology 1999;210:529-38.

14 Bittar RG, Olivier A, Sadikot AF, et al. Presurgical motor and somatosensory cortex mapping with functional magnetic resonance imaging and positron emission tomogranetic resonance imaging and pos
phy. $\mathcal{F}$ Neurosurg 1999;91:915-21.

15 Fandino J, Kollias SS, Wieser HG, et al. Intraoperative validation of functional magnetic resonance imaging and cortical reorganization patterns in patients with brain tumours tical reorganization patterns in patients with brain tumours involving

16 Lee CC, Ward HA, Sharbrough FW, et al. Assessment of functional MR imaging in neurosurgical planning. AfNR Am $\mathcal{F}$ Neuroradiol 1999;20:1511-19.

17 Woods RP, Cherry SR, Mazziotta JC. Rapid automated algorithm for aligning and reslicing PET images. f Comput Assist Tomogr 1992;16:620-33.

18 Yousry TA, Schmid UD, Alkadhi H, et al. Localization of the motor hand area to a knob on the precentral gyrus. A new landmark. Brain 1997;120:141-57.

19 Fox PT, Burton H, Raichle ME. Mapping human somatosensory cortex with positron emission tomography. 7 Neurosurg 1987;67:34-43.

20 Ganslandt O, Steinmeier R, Kober H, et al. Magnetic source imaging combined with image-guided frameless stereotaxy: a new method in surgery around the motor strip. Neurosura new method in sur.

21 Buchner H, Adams L, Knepper A, et al. Preoperative localization of the central sulcus by dipole source analysis of early somatosensory evoked potentials and threedimensional magnetic resonance imaging. I Neurosurg 1994;80:849-56.

22 Krings T, Chiappa KH, Cuffin BN, et al. Accuracy of electroencephalographic dipole localization of epileptiform activities associated with focal brain lesions. Ann Neurol 1998;44:76-86.

23 Krings T, Buchbinder BR, Butler WE, et al. Stereotactic transcranial magnetic stimulation: correlation with direct electrical cortical stimulation. Neurosurgery 1997;41:131926.

24 Frahm J, Merboldt KD, Hänicke W, et al. Brain or vein: oxygenation or flow? On signal physiology in functional MR of human brain activation. NMR Biomed 1994;7:45-53.
25 Lai S, Hopkins AL, Haacke EM, et al. Identification of vascular structures as a major source of signal contrast in high resolution $2 \mathrm{D}$ and $3 \mathrm{D}$ functional activation imaging of motor cortex at 1.5T: preliminary results. Magn Reson Med 1993:30:387-92.

26 Duvernoy HM, Delon S, Vannson JL. Cortical blood vessels of the human brain. Brain Res Bull 1981;7:519-79

27 Krings T, Erberich S, Roessler F, et al. MR BOLD signal differences in parenchymal and large draining vessels. mplications for functional MR Imaging. AfNR Am f Neuroradiol 1999;20:1907-14.

28 Boxerman JL, Bandettini PA, Kwong KK, et al. The intravascular contribution of fMRI signal change: Monte Carlo modeling and diffusion-weighted studies in vivo. Magn Reson Med 1995;34:4-10.

29 Boxerman JL, Hamberg LM, Rosen BR, et al. MR contrast due to intravascular magnetic susceptibility perturbations. due to intravascular magnetic suscep

30 Weisskoff RM, Zuo C, Boxerman JL, et al. Microscopic susceptibility variation and transverse relaxation: theory and experiment. Magn Reson Med 1994;31:601-10

31 Buchbinder BR, Cosgrove GR. Cortical activation MR studies in brain disorders. Magn Reson Imaging Clin N Am 1998;6:67-93.

32 Lee AT, Glover GH, Meyer CH. Discrimination of large venous vessels in time-course spiral blood-oxygen-leveldependent magnetic resonance functional neuroimaging. Magn Reson Med 1995;33:745-54.

33 Malonek D, Grinvald A. Interactions between electrical activity and cortical microcirculation revealed by imaging spectroscopy: implications for functional brain mapping. Science 1996;272:551-4.

34 Kim SG, Hendrich K, Hu X, et al. Potential pitfalls of functional MRI using conventional gradient recalled echo techniques. NMR Biomed 1994;7:69-74.

35 Gati JS, Menon RS, Ugurbil K, et al. Experimental determination of the BOLD field strength dependence in vessels and tissue Magn Reson Med 1997;38:296-302.

36 Duyn JH, Moonen CTW, vanYperen GH, et al. Inflow versus deoxyhemoglobin effects in BOLD functional MRI sus deoxyhemoglobin effects in BOLD functional MRI

37 Pawlik G, Rackl A, Bing RJ. Quantitative capillary topography and blood flow in the cerebral cortex of cats: an in vivo phy and blood flow in the cerebral cortex of cat:

38 Kwong KK. Functional MRI with echo planar imaging. Magn Reson Q 1995;11:1-20

39 Weisskoff RM. Functional MRI: are we all moving towards artifactual conclusions? Or fMRI fact or fancy? NMR Biomed 1995;8:101-103.

40 Hajnal J, Myers R, Oatridge A, et al. Artefacts due to stimulus correlated motion in functional imaging of the brain. Magn Reson Med 1994;31:283-91.

41 Strother SC, Anderson JR, Xu XL, et al. Quantitative comparisons of image registration techniques based on high-resolution MRI of the brain. 7 Comput Assist Tomogr 1994;18:954-62.

\title{
Narrative Based Medicine, An Interdisciplinary Conference
}

\author{
Research, Narrative, and Practice
}

A two day conference-Monday 3rd and Tuesday 4th September 2001

\section{Homerton College, Cambridge, UK}

\section{BM7 Publishing Group}

For full details contact: BMA/BMJ Conference Unit, Tavistock Square, London, WC1H 9JP Tel: +44 (0)20 7383 6819; fax: +44 (0)20 7383 6663; email: clyders@bma.org.uk. www.quality.bmjpg.com 